HIDING THE GUILLOTINE 



\title{
HIDING THE GUILLOTINE
}

\section{PUBLIC EXECUTIONS IN FRANCE, 1870-1939}

\author{
EMMANUEL TAÏEB \\ TRANSLATED BY \\ SARAH-LOUISE RAILLARD \\ FOREWORD BY \\ MITCHEL P. ROTH
}


The author and publisher would like to thank the Institut Universitaire de France for providing financial support for the translation of this book.

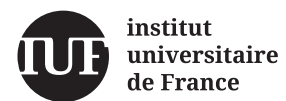

Original French edition (C) Éditions Belin/Humensis, La guillotine au secret: Les exécutions publiques en France, 1870-1939, 2011.

English language translation (C) Cornell University, 2020.

All rights reserved. Except for brief quotations in a review, this book, or parts thereof, must not be reproduced in any form without permission in writing from the publisher. For information, address Cornell University Press, Sage House, 512 East State Street, Ithaca, New York 14850. Visit our website at cornellpress.cornell.edu.

First published 2020 by Cornell University Press

Library of Congress Cataloging-in-Publication Data

Names: Taïeb, Emmanuel, author. | Raillard, Sarah-Louise, translator. | Roth, Mitchel P., 1953-, writer of foreword.

Title: Hiding the guillotine : public executions in France, 1870-1939 / Emmanuel Taïeb, translated by SarahLouise Raillard, foreword by Mitchel P. Roth. Other titles: Guillotine au secret. English

Description: Ithaca [New York] : Cornell University Press, 2020. Includes bibliographical references and index.

Identifiers: LCCN 2019053351 (print) | LCCN 2019053352 (ebook) | ISBN 9781501750946 (hardcover) | ISBN 9781501750953 (ebook) | ISBN 9781501750960 (pdf)

Subjects: LCSH: Capital punishment—France-History19th century. | Capital punishment-France-History20th century. | Guillotine-France-History19th century. | Guillotine-France-History20th century.

Classification: LCC HV8699.F8 T3513 2020 (print) | LCC HV8699.F8 (ebook) |

DDC 364.660944/ 09041—dc23

LC record available at https://1ccn.loc.gov/ 2019053351

LC ebook record available at https://lccn.loc.gov / 2019053352 
Civilization is on the easel.

_Victor Hugo, 1866 
